\title{
Relationship between Spacing Rasio and Stiffness Coefficient by Using Smoothed Particle Hydrodynamics Method to Simulate Water - Solid Interaction
}

\author{
Anisa Wulandari , R.R Dwinanti Rika , Jessica Sjah , and Herr Soeryantono \\ Civil Engineering Department, Faculty of Engineering, Universitas Indonesia, Depok, Indonesia
}

\begin{abstract}
Scouring Phenomenon directly occurs on materials due to the motion of water flow and water borne sediments that researchers in the world continue to investigate. Scouring are then continuously developed in Computational Fluid Dynamics (CFD) to be able to estimate scouring effects by analyzing interaction between fluid and solid. Water and solid interaction can be researched by realizing three dimensional numerical modeling (3D) using Smoothed Particle Hydrodynamics Method which is modeling and visualizing fluid behavior with a Lagrangian approach in particle scale (micro scale), a more particle approach realistic than the grid approach. Using this method, the results of each particle can be reviewed either by their property values or visually so that the results are obtained more representatives. One of the factors affecting fluid-solid modeling is spacing ratio between solid particle and fluid particle. To obtain the correct physical results, it is required to consider the influence of spacing ratio and the value of Stiffness Coefficient (Ks) needed.
\end{abstract}

\section{Introduction}

Definition of scouring in Sucipto (2004) [1] is the change of a stream accompanied by the transfer of material through the action of fluid motion. In other words, scouring phenomenon is a direct material grinding phenomenon that occurs due to the flow of fluid and the sediment carried by the fluid. The grinding of this material occurs in various domains such as agriculture, ecology, and engineering. Scouring phenomenon occurs in many civil infrastructure buildings involving water interaction with structural materials (soil or concrete) such as pier or abutment of bridges and dams.

There have been many studies done for depiction of scouring phenomena such as Lacey's Theorem method (1930), Raudkivi (1997), and Rijn (1989). However, the result of the process cannot be used visually because it only presented the mathematical model.

The simulation process that can distinguish water and soil layers realistically as a form of interaction between water and soil grains is crucial to perform numerical analysis on various problems. Finite Element Method (FEM) simulations typically do not cover the impact of water flow on the soil surface [2]. The case of scouring analysis due to the interaction between water and solid can be investigated by realizing the three dimensional numerical modeling by using Smoothed Particle Hydrodynamics (SPH) Method [3]. The SPH method was chosen to overcome the limitations of FEM. SPH method can be used to model and visualize fluid behavior with a more realistic particle approach than the grid approach as the researches that have been done by Straroszczyk
(2010), Kristof (2010), Sucipto (2004), Vetsch (2012), and Dalrymple, R. et al. (2015).

SPH method is a numerical method developed directly for dynamic fluid astrophysics in which current variables are defined on a set of moving points representing the constant period of incompressible particle [4]. The basic principle of the SPH method is related to the completion of the Navier-Stokes equation according to the variables required both internal and external variables. The advantages of this method can be used to assess the property of each particle and visually the results obtained more representative.

This paper is a preliminary study to model the interaction between fluid particles and solid particles present in the scouring phenomenon. To model the scouring phenomenon appropriately, an algorithm is required to provide an interaction between fluid particles and solid particles. The fluid-solid interaction algorithm is inspired by P. Kristof et al. (2009) [5] who have coupled the erosion simulation with a Lagrangian approach based on SPH Method. Some simulations have been carried out to make qualitative validations and to study some numerical parameters. This paper completed the research done to study the relation of numerical parameters between spacing ratio with stiffness coefficient (Ks) in order to get the modeling results that have correct physical meaning. Scouring phenomenon is represented by a collision process due to interaction between water-solid particles. The collisions between particles were regarded as perfectly elastic and the modeling process used FORTRAN program platform. The influence of chemical reactions is neglected. The Law of Conservation of Mass, the Law of Conservation 
of Energy, and the Law of Conservation of Momentum are applied.

\section{Literature Study}

\subsection{Smoothed Particle Hydrodynamics}

The SPH method is a meshfree, Lagrangian, particle method whose characteristics have advantages in the adaptive level of the approximate value of the property to the random distribution of the particles[6]. The stability, accuracy, and speed of the SPH method depend heavily on the choice of kernel smoothing type [7]. There are three kinds of kernels commonly used in calculating the SPH method of default kernel, Pressure Kernel, and Viscosity kernel. The SPH method is able to formulate the required variables as defined in the Navier-Stokes equation. The acceleration of the simulated particles is obtained from the division of both internal and external forces which are defined by the Navier-Stokes equation with the density.

The basic form of the Navier-Stokes equation in the Lagrangian formulation for fluids under incompressible and isothermal conditions is shown in Kelager (2003) [8]:

$$
\left(\frac{d u}{d t}+\nabla . u\right)=-\nabla p+\mu \nabla^{2} u+f^{\text {external }}
$$

Where is pressure gradient, is viscosity. The density of particle $\mathrm{i}$ can be formulated by the following equation:

$$
\rho_{i}=\sum_{j} m_{j} W\left(r_{i}-r_{j}, h\right)
$$

The internal force is a force whose source of work comes from within the water particle itself and it consists of pressure force and viscosity force.

$$
\begin{array}{r}
f_{i}^{\text {pressure }}=\sum_{j \neq i}\left(\frac{p_{i}}{2}+\frac{p_{j}}{2}\right) \frac{m_{j}}{\rho_{j}} \nabla W\left(r_{i}-r_{j}, h\right) \\
f_{i}^{\text {viscosity }}=\mu \sum_{j \neq i}\left(u_{j}-u_{i}\right) \frac{m_{j}}{\rho_{j}} \nabla^{2} W\left(r_{i}-r_{j}, h\right)
\end{array}
$$

The external forces are presented to balance internal forces consist of gravity,buoyancy, and surface tension.

$$
\begin{gathered}
f_{i}^{\text {gravity }}=\rho_{i} g \\
f_{i}^{\text {buoyancy }}=b\left(\rho_{i}-\rho_{0}\right) g \\
f_{i}^{\text {surface tension }}=-\sigma \frac{n_{i}}{\left\|n_{i}\right\|}\left(\sum_{j} \frac{m_{j}}{\rho_{j}} W\left(r_{i}-r_{j}, h\right)\right)
\end{gathered}
$$

\subsection{Fluid-Solid Interaction Algorithm}

The equation to handle the interaction is using the boundary approach which is proposed by Amada, T (2006) [9] and modified by P. Kristof (2009). This boundary approach is added into external force as boundary force $(\mathrm{fb})$. Therefore, the external force is the summation of the gravity, surface tension, buoyancy, and boundary forces. The Boundary force consists of Nonpenetration Force (fnp) and Non-Slip Force (fns).

$$
f^{b}=f^{n p}+f^{n s}
$$

The Non-Penetration force indicates that the fluid can not penetrate the boundary surface. The penalty-force method was used to repel the fluid particles from the boundary.

$$
f^{n p}=\left(K_{s} d-(\boldsymbol{v} . \boldsymbol{n}) K_{D}\right) \boldsymbol{n}
$$

Where $\mathrm{Ks}$ is the maximum force due to the stiffness present at the boundary and $\mathrm{Kd}$ is the damping coefficient for velocity of the incoming fluid particles (v) and the penetration distance. $\mathrm{n}$ is the normal line length. The nonslip force is the force where the fluid velocity at the modeling boundary is zero so non-slip condition occurs.

$$
f^{n s}(r)=\sum_{b} L_{b}^{2} \tau^{v i s c}\left(\left|r-r_{b}\right|\right)
$$

Where $\mathrm{Lb}$ is the distance between particles and $\tau$ visc is traction. The traction can be formulated as follows:

$$
\tau^{v i s c}(r)=-\mu \nu \nabla^{2} W_{v}(r, h)
$$

where $\mu$ is the friction constant which is at the boundary and $\mathrm{Wv}$ is the viscosity of the kernel.

The collision detection process is done by reviewing the position and velocity of the particles at each time-step. If the newest position of the particle can not provide enough information about the previous position, then the review process can be done by doing a flashback on the speed track.

The collision detection process is reviewed by analysis of the critical shear stresses of the solid material to the boundary style. In the simulation using the SPH method, it needs time integration process to obtain new position and speed value for each $t$. The time integration scheme to be used in this research is the Leap-Frog scheme.

\section{Research Methodology}

The computational domain that will be used in this research model is a rectangular parallel-piped box with dimensions for the $\mathrm{x}, \mathrm{y}, \mathrm{z}$ axes are $0.1 \mathrm{~m}, 0.1 \mathrm{~m}$, and 0.5 $\mathrm{m}$, respectively as shown in Figure 1 and Figure 2.

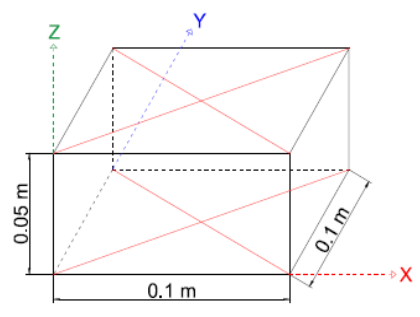

Fig. 1. Geometry of Computational Domain : Domain Model Drawing 


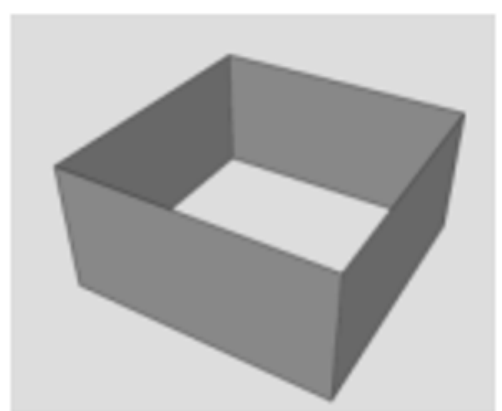

Fig. 2. Geometry of Computational Domain : Domain Model $3 \mathrm{D}$

\subsection{Numerical Parameter}

There are various initial numerical and physical parameters that have been determined before the modeling process was done. The initial physical parameters of fluid particles in the modeling were shown in Table 1. The solid particle density used in this study is $2800 \mathrm{~kg} / \mathrm{m} 3$.

Table 1. Initial Physical Parameters of Fluid

\begin{tabular}{|c|c|c|}
\hline Parameter & Value & Unit \\
\hline Rest Density $(\rho \mathrm{o})$ & 998,29 & {$[\mathrm{Kg} / \mathrm{m} 3]$} \\
\hline Buoyancy Diffusion $(\mathrm{b})$ & 0 & $\mathrm{n} / \mathrm{a}$ \\
\hline Viscosity $(\mu)$ & $1,0003 \mathrm{x}$ & {$[\mathrm{Pa} . \mathrm{S}]$} \\
& $10-3$ & \\
\hline Surface Tension $(\sigma)$ & 0,0736 & {$[\mathrm{~N} / \mathrm{m}]$} \\
\hline Gas Stiffness $(\mathrm{k})$ & 0,00001 & {$[\mathrm{~J}]$} \\
\hline
\end{tabular}

The independent variables that will be input in the model are the number of particles, the initial velocity of the particles, the number of desired iterations, stiffness coefficient value $(\mathrm{Ks})$, damping coefficient value $(\mathrm{Kd})$, and time step. In this modeling, 500 iterations with $0.01 \mathrm{~s}$ time step will be used to achieve the stable condition. In addition to the variables mentioned, such as the value of friction at 0.1 is taken from a case study that was conducted by Vestch (2012) [10]. The influence of Ks and $\mathrm{Kd}$ values is will be analyzed in the modeling of solid particles. The dependent variable is density, speed and pressure. There is also an evaluation parameter such as Law of Energy Conservation and Law of Mass Conservation which has been inputted also on the SPH modeling algorithm. It is expected that the review variable can behave in accordance with the law that has been determined.

Numerical Simulation. Three cases of modeling scenarios will be performed in this research: Rain Water Concrete Phenomenon (Model 1), Water - Concrete Phenomenon (Model 2), Water - Granular Solid Phenomenon (Model 3) as shown in Figure 3. The fluid particles are presented in blue color and the solid particles are presented in red color. Each phenomenon will be studied by varying the magnitude of the stiffness coefficient (Ks) and the damping coefficient $(\mathrm{Kd})$ of the solid material.

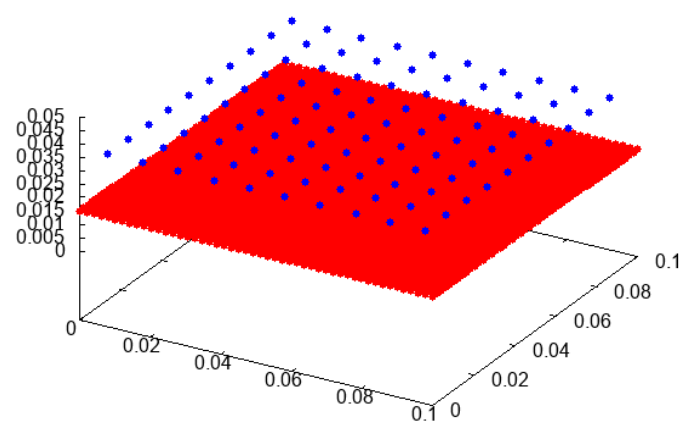

(a)

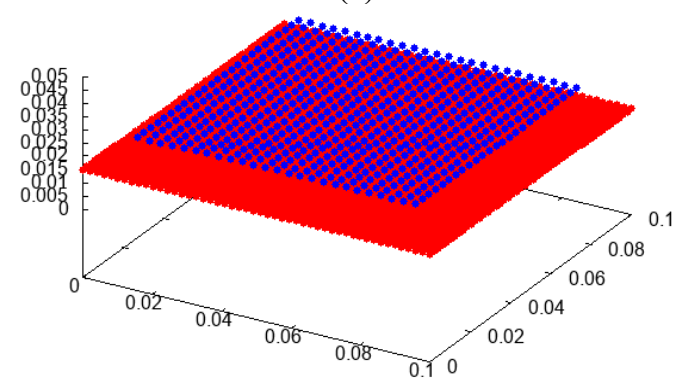

(b)

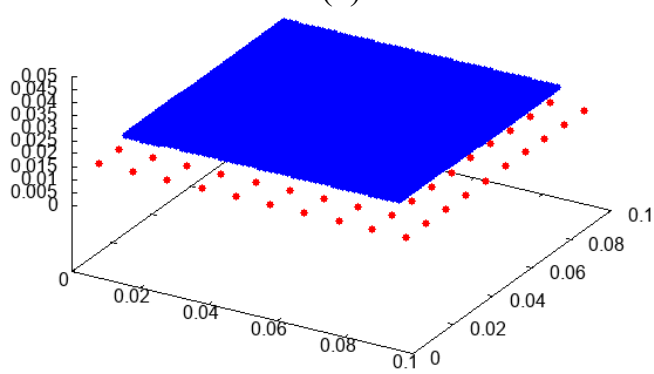

(c)

Fig. 3. Initial condition of: (a) Model 1; (b) Model 2; (c) Model 3

Model 1 used 100 fluid particles and 2500 solid particles where the distance between fluid particles is $0.01 \mathrm{~m}$ with the spacing ratio of solid and fluid particles is 0.20 (Figure 3(a)). Model 2 used 625 fluid particles and 2500 solid particles where the distance between fluid particles is $0.0033 \mathrm{~m}$ with the spacing ratio of solid and fluid particles is 0.61 (Figure 3(b)). Model 3 using 2500 fluid particles and 100 solid particles where the distance between fluid particles is $0.00204 \mathrm{~m}$ with the spacing ratio of solid and fluid particles is 4.902 (Figure 3(c)).

\section{Results and Analysis}

The analysis will be performed to determine the relationship between fluid distances and solid particles (spacing ratio) with the variation value of stiffness coefficients $(\mathrm{Ks})$ to obtain correct physical behavior model. 
Table 2. Result of Fluid-Solid Interaction with $\mathrm{Ks}=50,000$

\begin{tabular}{|c|c|c|c|}
\hline Model & Model 1 & Model 2 & Model 3 \\
\hline $\begin{array}{c}\mathrm{Ks}= \\
50,000\end{array}$ & & & \\
\hline
\end{tabular}

By using Ks equal to $5,000 \mathrm{~N} / \mathrm{m}$, the fluid particles can not recognize the solid particles for all models. Meanwhile, for Ks equal to $50,000 \mathrm{~N} / \mathrm{m}, 76 \%$ and $99.68 \%$ of fluid particles in Model 1 and Model 2 respectively were able to recognize the solid particles, while the rest of fluid particles passed through the solid particles (Table 2). In Model 3 with Ks equal to 50,000 $\mathrm{N} / \mathrm{m}$, the fluid particles are able to recognize the solid particles entirely. To improve the quality of result, the value of Ks is enlarged up to $100,000 \mathrm{~N} / \mathrm{m}$ so that all the fluid particles can recognize the solid particles.

The calculation of the boundary forces for each model can be seen in Figure 4. It showed that the value of stiffness coefficient is directly proportional to the value of boundary force for each model with different spacing ratio variation.

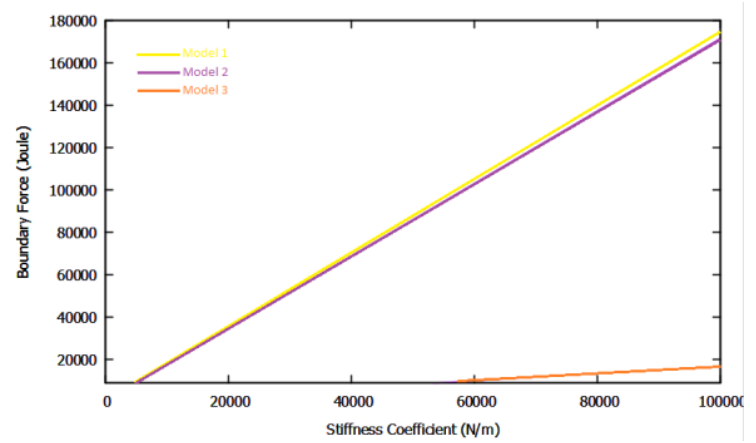

Fig. 4. Graphic of Average Boundary Force for each model

\section{Conclusion}

The way to model and analyze the interaction between solid and fluid due to fluid particle collision responses with solid particle resistance can be done using the Smoothed Particle Hydrodynamics (SPH) numerical approach. Based on the modeling and analysis that has been done, it is known that the value of stiffness coefficient (Ks) is directly proportional to the value of the boundary force of each particle which is affecting the ability of solid particles to recognize the fluid particles.

The main component of the Boundary force that influences the fluid response in the event of a collision is influenced by the value of Ks. The more rigid a particle, the value of its stiffness coefficient will be greater, so it will enlarge the boundary force. The boundary force contributes in determining the subsequent total force for fluid behavioural observation. The smaller value of Ks can cause the inability of fluid particles to recognize solid particles.

When associated with the spacing ratio between solid particles and fluid particles, it can be argued that the greater the spacing ratio the greater the value of $\mathrm{Ks}$ required for the fluid particles to be completely retained by the solid particle sequence.

There should be appropriate selection of spacing ratio and stiffness coefficient to obtain the results that match the actual physical conditions when the water particles are able to recognize solid particles.

This work is supported by Hibah PITTA 2018 funded by DRPM Universitas Indonesia No. 5000/UN2.R3.1/HKP.05.00/2018.

\section{References}

1. Sucipto, Pengaruh Kecepatan Aliran Terhadap Gerusan Lokal Pada Pilar Jembatan Dengan Perlindungan Groundsill, pp. 34. (2004)

2. G.R. Liu, M.B Liu, Smoothed Particle Hydrodynamics: A Meshfree Particle Method, Singapore: World Scientific Publishing. (2003)

3. B. Stefanova, K. Seitz, J. Bubel, J. Grabe, ICSE6 Paris. Water Solid Interaction using Smoothed Particle Hydrodynamics. (2012)

4. S. Sibilla, ERCOFTAT Bulletin, SPH Simulation of Local Processes, 41-44, (2007)

5. P. Kristof, et al., Hydraulic Erosion Using Smoothed Particle Hydrodynamics, Czech Republic - Purdue University, USA (2009)

6. G.R. Liu, M.B Liu, Smoothed Particle Hydrodynamics: A Meshfree Particle Method, Singapore: World Scientific Publishing. pp. 26-27 (2003)

7. M. Müller, D. Charypar, \& M. Gross, Particle-Based Fluid Simulation for Interactive Applications. Eurographics, SIGGRAPH Symposium on Computer Animation, (2003)

8. M. Kelager, Lagrangian Fluid Dynamics Using Smoothed Particle Hydrodynamics, Copenhagen, Denmark: Departement of Computer (2006) 
9. Amada, T. Real-time particle-based fluid simulation with rigid body interaction. In Game Prog. Gems 6, Charles River Media, pp. 189-205. (2006)
10. D. Vetsch, VAW, Numerical Simulation of Sediment Transport with Meshfree Methods (2012) 